\title{
Significados de la adolescencia en los relatos de una muestra de adolescentes y sus padres en la ciudad de Medellín*
}

Meanings of adolescence in the narratives of a sample
of adolescents and their parents in the city of Medellin

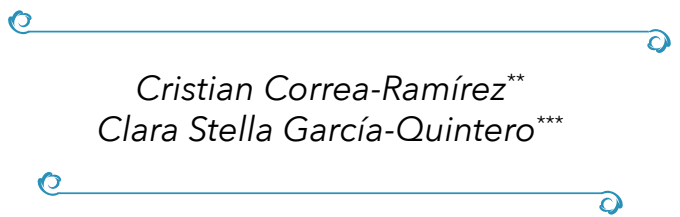

Recibido 12.07. 2019• Arbitrado 25. 08. 2019 •

Aprobado 20.09. 2019

Producto derivado de la investigación "Narrativas de adolescentes y sus padres en torno al riesgo en la adolecía" realizada entre los años 2014-2016 en el marco de la Maestría de Terapia Familiar y de Pareja de la Universidad de Antioquia.

** Psicólogo, Magister en Terapia familiar y de pareja, Terapeuta Familiar y de Pareja, Docente UPB -Medellín, Colombia. ORCID: 0000-0002-32176663, cristian.correa@upb.edu.co

*** Psicóloga, Magister en Terapia familiar y de pareja, Terapeuta Familiar y de Pareja, Docente Corporación Universitaria Minuto de Dios-Uniminuto - Seccional Antioquia- Chocó, Colombia. ORCID: 0000-0003-4087-3159, cgarciaqui1@uniminuto.edu.co

\section{Resumen}

Objetivo: Describir los significados de los adolescentes y sus padres, pertenecientes a una muestra seleccionada en la ciudad de Medellín, en torno a la adolescencia. Metodología: Se realizó una investigación cualitativa con enfoque fenomenológico de tipo narrativo. Se realizaron 7 entrevistas con adolescentes y 7 con los padres. Resultados: La adolescencia es vista por los padres y adolescentes desde 4 dimensiones: los cambios, las búsquedas, ser dueño del mundo y la exploración. Conclusión: La adolescencia se concibe por los participantes del estudio como una época de transición, tensiones y retos que favorecen el proceso de individuación.

Palabras clave: adolescencia, familia, individuación, tensiones familiares 


\section{Abstract}

Objective: To describe the meanings that adolescents and their parents attribute to adolescence. Methodology: A qualitative study was conducted with phenomenological hermeneutical approach, narrative research. 7 interviews with teenagers and 7 interviews with parents were conducted.Results: Adolescence is seen by parents and adolescents from 4 dimensions: changes, searches, owners of the world and exploration. Conclusion: Adolescence is seen as a time of transition, tensions and challenges that favor the process of individuation.

Key words: adolescence, exploration, tensions, changes, individuation.

\section{Introducción}

Este artículo se presenta en el contexto de la investigación "Narrativas del adolescente y sus padres en torno al riesgo en la adolescencia". La adolescencia ha sido tema de reflexión de diversos autores, conceptualizándola con distintos matices; sin embargo, son pocas las investigaciones que apuntan a comprender los significados que va adquiriendo la adolescencia desde el relato mismo de los adolescentes como sujetos de saber, en esa perspectiva la comprensión de la adolescencia va hilando ejes históricos, culturales y antropológicos que introduce dinamismo y la necesidad de una continua reflexión (Graff, 1999).A su vez, se establece un camino dialógico del escenario familiar en el que se incluye también el relato de padres sobre el significado que otorgan a ésta.

La adolescencia fue objeto de investigación científica desde el siglo XIX y muchos de estos estudios se consolidan durante la década de los 60 del siglo $\mathrm{XX}$. A la par que se abría paso la investigación y comprensión de la adolescencia, aparecían fenómenos donde se empezaban a tipificar distintas prácticas de riesgo vinculadas al adolescente: accidentes automovilísticos, enfermedades de transmisión sexual, alcohol, cigarrillos y sustancias psicoactivas (Grinder, 2001). La década de los 60 fue fundante para el surgimiento del imaginario social y científico del adolescente como sujeto de riesgo, revestido de distintos tintes negativos que pareciera perdurar en la actualidad (González y de la Hoz, 2011; Holguín, Cubillas, Pérez, Valdés, 2007; Fuentes, Alarcón, García, F. y Gracia, E., 2015; Varela, Salazar, Cáceres y Tovar, 2007).

La comprensión de la adolescencia como una etapa de riesgo ha propiciado un estereotipo negativo de los adolescentes, ya en 1964 Bandura (1975) 
llamaba la atención del lugar de los medios de comunicación y la academia, en presentar al adolescente como problemático y delincuente, etiquetando a todo un grupo poblacional, asunto que el autor denominó como profecía instigadora de la adolescencia. La generalización de la adolescencia desde una mirada problemática es un fenómeno presente reforzado por los medios de comunicación, quienes en el afán de dar una primicia informativa privilegian el sensacionalismo sobre el rigor informativo (Carvajal, 2010).

Si se reitera continuamente en los medios de comunicación y en la sociedad en general una imagen del adolescente como sujeto rebelde, descarrilado, no será extraño que el adolescente termine aceptando y viviendo bajo dichos calificativos, fomentando una imagen sesgada de estos (Suárez, Del MoralyMusitu, 2013). En contraste, se puede encontrar en los adolescentes una búsqueda de autonomía en contextos sociales donde no se les considera listos para asumir su independencia; mostrando que el fenómeno va más allá de las actividades percibidas socialmente como negativas (Omatseye, 2007).

Otros autores, como Michaud (2006), señalan que los relatos de los adolescentes, como agentes de problemas, han deteriorado la relación entre las generaciones. Muchos de los comportamientos vistos como peligrosos en la adolescencia obedecen a un momento de exploración y conocimiento que no necesariamente comprometen la integridad del adolescente (Coleman y Hendry, 2003); por lo tanto, el concepto de las prácticas de riesgo se reserva a las situaciones en las que, los adolescentes se involucran en un uso excesivo de alguna práctica, como el consumo del alcohol y de cannabis (Michaud, 2006).

De otro lado, la adolescencia en términos etarios ha tenido multiplicidad de criterios. Así, por ejemplo, Carrasco (2006) clasifica la adolescencia en temprana (10 a 13 años), media (14 a 17 años) y tardía (17 a 19 años), mientras que Aberastury y Knovel (2014) propone la adolescencia entre los 13 y 21 años, haciendo distinción de género: en las mujeres la adolescencia va desde los 12 y se extiende hasta los 21 años y en los varones de los 14 a los 25 años. Teniendo en cuenta las diferencias que se pueden encontrar, se toma como referencia para esta investigación, las consideraciones que establece la ley de infancia y adolescencia para Colombia, que en su artículo 3 define a los adolescentes como las personas menores de 18 años y que se encuentran entre los 12 y los 18 años de edad (Ley 1098, 2006).

La adolescencia vista ya no como una categoría etaria sino como fenómeno, es definida por Aberastury y Knobel(2014) como "periodo de transición entre la pubertad y el estadio adulto del desarrollo y que en las diferentes sociedades este período puede variar como varía el reconocimiento de la condición adulta que se le da al individuo" (p.38). Lo que está en juego en esta etapa 
de la vida es el proceso de individuación y desprendimiento, por lo que la adolescencia debe ser vista como un proceso de cambio que se da de manera universal, siendo favorecido o dificultado por cada cultura.

Continuamente estos autores hacen referencia a la adolescencia como un periodo de múltiples duelos, la aceptación y construcción de una nueva identidad; identifican allí tres tipos de duelos: el primero, por el cuerpo infantil, el segundo, por el rol y la identidad infantil, y el tercer duelo, por los padres de la infancia (Aberastury y Knobel, 2014).

La adolescencia está vinculada al desarrollo de un proceso de individuación, la conquista de la autonomía más allá del orden familiar supone que en cada cultura se asuma unas particularidades (Aberastury y Knobel, 2014; Blos, 2011; Omatseye, 2007). Las dificultades en la comprensión de dicho proceso han propiciado que rápidamente se etiquete al adolescente bajo una perspectiva de crisis y de problemático.

En este sentido, es relevante presentar desde la perspectiva del adolescente y sus padres, los significados que dan a la adolescencia, toda vez que son los protagonistas; agentes activos en este campo relacional. Así, se obtiene una conceptualización situada en el escenario familiar que busca aportar tanto en el ámbito académico como fuera de él. Su novedad se instaura en traer la voz del adolescente como sujeto competente al tiempo que se posibilita el dialogo con los significados que algunos padres otorgan a la adolescencia.

\section{Método}

La investigación se desarrolla con enfoque cualitativo, de método fenomenológico - hermenéutico.

\section{Participantes}

Los participantes en la investigación fueron siete adolescentes entre los catorce y dieciocho años y siente padres (seis madres y un padre), uno por cada participante. Los participantes fueron elegidos de manera intencional, por conveniencia, de acuerdo con los objetivos de la investigación. Para la selección de los participantes se envió invitación a diferentes colegios de la ciudad de Medellín, considerando diferentes estratos socioeconómicos. Hicieron parte de la presente investigación los adolescentes y padres quienes estuvieron interesados en participar, de manera voluntaria. Se destaca que, de las siete familias participantes, cinco pertenecen a estrato socio económico medio y dos a estrato socio económico alto. 


\section{Instrumentos}

Se utilizó la entrevista semiestructurada como instrumento para la recolección de la información, ésta fue elaborada a partir de las categorías: Significados: ideas o conceptos que representan o evocan los elementos lingüísticos asociados al riesgo y la adolescencia; Prácticas: entendida como aquellas conductas o acciones que reconoce el adolescente y su familia en torno al riesgo y, Relaciones: correspondencia o conexión que hay entre las prácticas de riesgo y las vivencias del adolescente y su familia. Este artículo proviene exclusivamente de los significados construidos por los adolescentes y padres participantes, sobre adolescencia.

Inicialmente el instrumento fue validado a través de, la revisión por pares expertos y, en un segundo momento, a través de la aplicación de una entrevista piloto en el que se identificó las características generales de las categorías objeto de interés de la investigación.

\section{Procedimiento}

Para contactar a los participantes se envió comunicación escrita a colegios de la ciudad, pertenecientes a diferentes estratos socioeconómicos. Una vez se tuvo la aprobación de las directivas de las instituciones se procedió con el contacto con los estudiantes y sus padres. En los colegios pertenecientes a estratos socioeconómico 6 se realizó a través de correo institucional, en los demás colegios, pertenecientes a estratos socioeconómicos 2 y 3 se realizó de manera personal.

Los adultos participantes en la investigación firmaron consentimiento informado y los adolescentes, asentimiento informado. Ambos aprobados por el comité de ética del departamento de psiquiatría de la Universidad de Antioquia.

Las entrevistas fueron realizadas por separado a los padres y a los adolescentes, en la mayoría de ellas estuvieron presentes, al mismo tiempo, los dos investigadores. El promedio de duración de cada entrevista fue de 60 minutos.

\section{Análisis de información}

Cada una de las entrevistas fue transcrita, generando un código de identificación, de acuerdo al número de entrevista efectuado, Entrevista 1, E2, E3 y a la población participante: AH-Adolescente hombre, AM-adolescente mujer, P-padre, M-madre. Dado que la investigación buscó la participación 
de familias de distintos estratos socios económicos, también aparece este elemento dentro de la codificación, por ejemplo: E1, AM, 3-entrevista 1, adolescente mujer, estrato 3; E1, M, 3: entrevista 1, madre, estrato 3.

Posteriormente las entrevistas fueron analizadas a través del programa Atlas ti 6, para esto se crearon códigos de corte inductivo, los cuales fueron agrupados durante el proceso en categorías más amplias proceso deductivo. Se generaron memos analíticos con cada una de las categorías principales, dicha información se convirtió en insumo básico para la comprensión de los relatos de padres y adolescentes.

\section{Resultados}

\section{Significados de la adolescencia}

Los resultados encontrados, graficados en la Figura 3, muestran cuatro categorías en la forma como se concibe la adolescencia: como época de cambios, como búsqueda, el adolescente dueño del mundo y el adolescente como explorador.Las dos primeras categorías son comunes entre padres y adolescentes, la tercera se recoge los relatos de los padres, y en la cuarta lo expresado por los adolescentes.

Figura 3: Significados de adolescencia

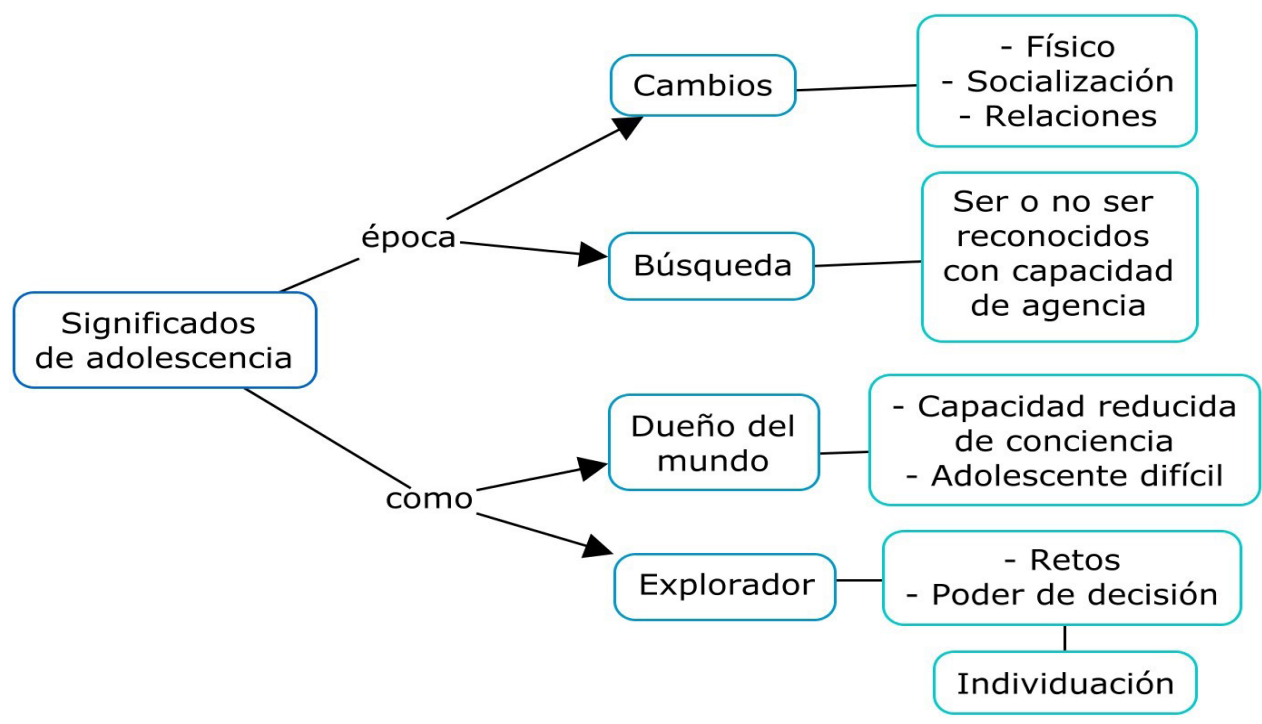

Fuente: Elaboración propia 


\section{La adolescencia como época de cambios}

Los padres y adolescentes definen la adolescencia como una época de cambios, en los que destacan: biológicos, relacionales, cognitivos, emocionales, familiares, espaciales.

La adolescencia sí, es eso, que quieren estar en combitos, que una cosa que la otra, que, en bailes, que en farras (E6M3).

A ver, pues la adolescencia es en sí un cambio hormonal en el cuerpo, que ya a partir de los 10 años va comenzando a tener sus cambios en el cuerpo (E5M2).

Porque a usted le empieza a gustar los muchachos, me gustó fulanito, peranito, uno tiene que saber controlar eso; porque no es lo mismo mirar una persona que la atrae a entablar una relación, ya como dicen ahora novios o amigovios, yo le explicaba mucho eso a la niña (E5M2).

Uno antes mantenía jugando en la calle, pues ahora uno ya no juega así como antes, uno va a fiestas, mantiene con los amigos, uno antes jugaba con muñecas, ya no, pues, mucho cambia (...) cuando uno era chiquito a uno no le importaba casi nada, si uno se aporreaba uno sí lloraba pero seguía jugando y pues uno peleaba con un amiguito y al rato volvían a hablarse en cambio ya ahora no, ya uno se enoja con un amigo y uno se demora mucho tiempo en volverse a hablar por ser orgulloso (...) [el adolescente] es joven, es alegre,le gusta salir y experimentar(E5AM2).

Las transformaciones del adolescente son un primer escenario que da cuenta de los significados que rodean la adolescencia; la fiesta emerge como escenario relacional, vinculado a los amigos, los espacios de socialización comienzan a vivirse con frecuencia fuera de los espacios familiares. A su vez, no se habla de cambios sin percibir malestar y tensión en el binomio relacional padres-adolescente, fenómenos que expresan en sí mismo una búsqueda de autonomía por este y un intento de control por parte de los padres.

\section{La adolescencia como búsqueda: entre ser y no ser}

Una de las nociones que más se repite en los relatos de los padres, ubica al adolescente como un sujeto que no sabe dónde está, en contraste, el adolescente se define desde una búsqueda continua y permanente por ser, por encontrarse a sí mismo. 
En el relato de los padres entrevistados, el adolescente no es un adulto, pero sus acciones pueden estar en dicha vía; quieren tener una vida de adultos. Se aprecia que parte de la búsqueda que los padres le otorgan al adolescente está en dicho camino, no obstante, para estos, el adolescente todavía no está listo o preparado para llevarla.

La adolescencia es un no saber dónde está, o sea no soy niño, pero tampoco soy grande, pero quiero ser grande y no puedo ser grande, (...) una cantidad de cosas que no, es ese no saber y no estar en ningún sitio específicamente, sino es tratando de ser y todavía no son. (E3M6)

En contraste, los adolescentes, aunque reconocen que no están propiamente inscritos en el mundo de los adultos y tampoco lo están en el mundo de la infancia, identifican en esta etapa un momento de nuevas búsquedas, de autodescubrimiento, de autoconstrucción, un momento generador clave en el devenir de su existencia.

[la adolescencia es] la etapa donde uno se encuentra a sí mismo (...) pero cuando uno en verdad se rige, cuando uno en verdad se encuentra a sí mismo en la adolescencia (...) uno ya tiene que decir como más o menos qué le va gustando, uno tiene que ir decidiendo qué va a ser cuando sea grande, uno tiene que ir viendo sus fortalezas y sus dificultades para hablarlas con la gente, para superarlas, es prácticamente eso, es el momento de la vida donde uno prácticamente no es nadie, uno ya está muy grande para ir a los jueguitos de diversiones pero todavía está muy pequeño para ir a las discotecas, uno está ya muy grande como para molestar ya en la buseta, pero está muy pequeño como para manejar todavía, entonces es el momento intermedio donde se rige lo que va a ser cuando sea grande, como el destino de esa persona (E4AH6).

Definirse en el terreno de la nada introduce un nivel de incertidumbre en el adolescente; pareciera, que esta condición lo impulsara en un escenario de búsquedas y nuevos encuentros, en un terreno de vacío fértil. Se construye experiencias a partir de espacios poco habitados en la infancia, se le otorga a la decisión un peso importante que marca la propia identidad.

\section{El adolescente dueño del mundo en contraste con el adolescente explorador}

El adolescente dueño del mundo es una visión de los padres, en la que perciben en los adolescentes poca noción de los peligros, así como una capacidad 
reducida de la conciencia, esta visión también habla de un adolescente que pareciera inmortal y que es difícil. De otro lado, los adolescentes se conciben como exploradores. En sus relatos, se destaca la adolescencia como una posibilidad de exploración, llena de retos, con poder de decisión.

Son cosas que yo digo no tienen noción de peligro, son inmortales, un adolescente no cree que se puede matar, la mayoría, no voy a ser de todos o de ninguno, muchos creen que no mueren, entonces hacen conductas suicidas de ese tipo (E4M6).

Adolescente [es una] persona [de] 13 años en adelante, que pasa por cambios hormonales y empieza a tener una nueva visión de su vida y del mundo con forme a lo que se ha basado y ha aprendido (...) uno ya tiene que empezar a hacer muy bien las cosas, que uno ya tiene que hacer ya una mentalidad de su propia vida (...) por ejemplo yo que pienso estudiar algo en una universidad, no sé qué, con qué medios lo voy a pagar, yo no sé, pero para mí yo tengo que empezar como qué voy a hacer, cómo voy a organizar mi tiempo, cómo voy a organizar mis estudios, el tiempo con mis amigos, con mi novia y todo eso(E6AH3).

Se observa entonces que los adolescentes reconocen la adolescencia como un momento de cambios donde se da lugar a la exploración, es un momento en el que se reconocen retos y oportunidades bajo diferentes vías; en contraste con los padres, no refieren expresiones en las que no se reconozca el peligro, incluso aceptan sus limitaciones y reconocen que están en un proceso de madurez.

Pese la visión que tienen los padres de la adolescencia en sus hijos, la reflexión que introducen sobre sus propias vivencias construidas en esta etapa destaca el enfrentarse a la vida misma, explorar más allá de los límites familiares; la muerte de sus propios padres, las dificultades familiares, económicas, convocaron distintos desafíos.

Me parece curioso que mencione los catorce, porque digamos que a esa edad yo me enfrenté a las decisiones más difíciles por las cuales yo tuve que pasar en mi vida, que fue la muerte de mi papá.Mi papá falleció cuando yo tenía catorce años (E2P3).

Mi adolescencia se basó prácticamente en salir a la calle, y mirar qué iba a hacer con mi vida, porque mi mamá me faltó a los 17, mi papá no asumió la responsabilidad que tenía que asumir como padre de familia, y ¿qué hice?, pues salir a la calle, lucharla, trabajar para poder estudiar en la nocturna y poder terminar mi bachillerato (E1M3). 
De esta manera, coexiste en los relatos de los padres, una mirada de la adolescencia como una etapa difícil; pero al mismo tiempo, al mirar retrospectivamente al pasado, sus relatos se acercan a las formas como sus propios hijos la conciben; sin embargo, esto no logra ser integrado en sus relatos, incluso algunos le dan un tinte heroico a esta etapa vivida, pero en lo actual la conciben en sus hijos con matices negativos.

\section{Discusión}

Al indagar sobre los significados de la adolescencia en la voz misma del adolescente y sus padres, se encontró que parte de lo que sucede desde los relatos son historias de un transitar que va siguiendo el adolescente hacia la individuación. Para autores como Blos (2011), el camino a la individuación es un proceso que está presente en la adolescencia, este tiene varias implicaciones y modificaciones en la estructura interna y social del adolescente, pero ¿cómo se allana este camino a la individuación? Es común encontrar en los relatos de los participantes un reconocimiento de cambios en el adolescente: relación con los padres, relación con los amigos, cambios físicos, emocionales; estos cambios se asocian a una serie de duelos, en los que destaca duelo por el cuerpo infantil, duelo por el rol y la identidad y duelo por los padres de la infancia (Aberastury y Knobel, 2014).

Las transformaciones que acontecen en esta etapa invitan a establecer una nueva mirada del adolescente, en la que se reconozca y normalice, si se llega a presentar, las tensiones y conflictos emocionales o familiares que puedan acontecer como proceso de ajuste (OMS, 1999; Retuerto, 2002; Oliva et al., 2010).

Al finalizar la adolescencia se suele identificar un terreno distinto en las formas relacionales de padres e hijos que puede estar marcada por la reducción de tensión en la relación, en un escenario donde el padre ha reconocido y aceptado los cambios y elecciones del adolescente, pero, a su vez, este último camina en una vía de responsabilización de dichas elecciones (Omatseye, 2007).

Ahora bien, este no es un camino estandarizado para los adolescentes, los cambios y los procesos se van dando con matices, diferencias culturales y sociales (Aberastury y Knobel, 2014; Omatseye, 2007); la forma como se lleva a cabo la individuación puede ser favorecida o puede estar cargada de dificultades (Batista et al, 2006; Pallavicini, 2008; Nicholls, 2011; Cetina y Moreno, 2015; Uribe,; Sanabria, Orcasita y Barreto, 2016;). Los padres, por ejemplo, refieren la adolescencia como una etapa difícil y cargada de rebeldía; sin em- 
bargo, quedarse en un plano meramente descriptivo como una etapa difícil, o de rebeldía, impide ver pautas que conectan los discursos de los padres con la propia visión del adolescente (Janin, 2005; Omatseye, 2007).

Estos mismos padres refieren que su propia adolescencia fue un momento donde se les arrojó a la vida, sin aviso alguno; los padres se narraban como protagonistas, que tomaron diversas decisiones, exploraron una serie de caminos convertidos hoy en historias con un tinte de heroísmo, ¿acaso esa suerte de rebeldía se requiera entonces para ir más allá de los límites familiares?Quizás una de las formas en las que se puede propiciar nuevos enfoques en la concepción de la adolescencia, esté referida a la necesidad de propiciar nuevos espacios de reflexión en los que distintas generaciones puedan dialogar en torno a los aspectos en común que se han compartido frente a ésta.

Por ende, además de ser una etapa llena de retos, también es una etapa donde se explora más allá de los límites o discursos familiares, en la que se favorece la autonomía o la aparición de nuevos espacios que posibilitan el fortalecimiento de la propia identidad. Finalmente, esta mirada podría propiciar un escenario relacional de diálogos entre padres y adolescentes distintas, en las que se pueda tramitar temores, ansiedades y preocupaciones, desde una óptica donde se reconoce al adolescente como sujeto competente.

\section{Referencias}

Aberastury, A., Knobel, M. (2014). La adolescencia Normal. México D.F: Paidós Educador.

Bandura, A. (1975). Análisis del aprendizaje social de la agresión. En A. Bandura, \& E. R. Iniesta, Modificación de conducta. Análisis de la agresión y la delincuencia (págs. 307-347). México: Trillas.

Batista, J., García, M., Rodríguez, G., Máiquez, M., Martínez, M., Martin, J., Rodrigo, M.(2006). Estilos de vida en la adolescencia y su relación con los contextos de desarrollo. Cultura y educación: revista de teoría, investigación y práctica, 18(3-4), 381-396. https://doi.org/10.1174/113564006779173064

Blos, P. (2011). La transición adolescente (Tercera ed.). Argentina: Amorrortu.

Canales, M. (2006). Metodología de la investigación social. Santiago de Chile: LOM.

Carrasco, E. (2006). Terapia familiar orientada al desarrollo adolescente. En A. Roizblatts (Ed.), Terapia familiar y de pareja (págs. 426-434). Santiago, Chile: Mediterráneo. 
Carvajal, C. A. (2010). La creación mediática de los imaginarios colectivos sobre las drogas. FISCAM (Ed.), Observatorio de drogas, de Castilla, la Mancha, Jóvenes, Drogas y Comunicación, 6,13-26, http://www.od.jccm.es/admin/modulos/ publicaciones/pdf/118_3385yt04.pdf

Cetina, C., Moreno, J. (2015). Adaptación en adolescentes que practican actividad física frente a los que no practican. Informes Psicológicos, 15(1), 31-46. DOI: http://dx.doi.org/10.18566/nfpsicv15n1a02

Coleman, J., Hendry, L. (2003). Psicología de la adolescencia. Madrid: Ediciones Morata.

Flick, U. (2007). Introducción a la investigación cualitativa (Segunda ed.). Madrid: Ediciones Morata.

Fuentes, M., Alarcón, M., García, F., Gracia, E. (2015). Consumo de alcohol, tabaco, cannabis y otras drogas en la adolescencia, Anales de psicología, 31 (3), 1000-1007. http://dx.doi.org/10.6018/analesps.31.3.183491.

González, J., De la Hoz, F. (2011). Relaciones entre los comportamientos de riesgo psicosociales y la familia en adolescentes de Suba. Revista Salud Pública, 13 (1), 67-78. https://revistas.unal.edu.co/index.php/revsaludpublica/article/ view/33530/38088

Graff, H. (1999). Interdisciplinary explorations in the history of children, adolescents, and youth for the past, present a future. The Journal Of American History, 85(4), 1538-1547. DOI: $10.2307 / 2568273$

Grinder, R. (2001). Adolescencia. México D.F: Limusa.

Holguín, J., Cubillas, M., Pérez, R., Valdez, E. (2007). Intentos de Suicidio en adolescentes de educación media superior y su relación con la familia. Psicología y Salud, 17(1); 45-51. http://www.redalyc.org/pdf/291/29117105.pdf

Janin, B. (2005). Los padres, el niño y el analista: encuentros y desencuentros. Cuestiones de infancia, 9, 15-32. http://dspace.uces.edu.ar:8180/xmlui/ bitstream/handle/123456789/204/Los_padres_el_ni\%C3\%B1o_y_el_analista. pdf? sequence $=1$

Ley 1098. (2006). Diario Oficial 46446. Bogotá, Colombia.

Martínez, J. (1997). Desarrollo personal, ambiente familiar y relaciones de pareja en la adolescencia. Revista de psicología social, 12(1), 59-78.

Michaud, P. (2006). Adolescents and risks: Why not change our paradigm? Journal of Adolescent Health, 38, 481-483. DOI: 10.1016/j.jadohealth.2006.03.003 
Nicholls, E. (2011). Proceso de individuación en adolescentes con consumo problemático de drogas desde la mirada sitémico relacional. De familias y terapias, 20(31), 9-25. https://dialnet.unirioja.es/ejemplar/298158

Oliva, A., Rios, M., Antolín, L., Parra, A., Hernando, A., Pertegal, M. (2010). Más allá del déficit: Construyendo un modelo de desarrollo positivo adolescente. Infancia y aprendizaje, 33(2), 1-12, DOI: 10.1174/021037010791114562

Omatseye, B. (2007). The adolescent quest for autonomy: renegotiating a cordial relationship. College Student Journal, 41 (3), 623-630. https://eric. ed.gov/?id=EJ777973

Organización Mundial de la Salud. (1999). Programación para la salud y el desarrollo de los adolescentes. Ginebra: Organización Mundial de la Salud. https://www. who.int/maternal_child_adolescent/documents/trs_886/es/

Organización Mundial de la Salud. (2000). ¿Qué le ocurre con los muchachos? Suiza: Organización mundial de la salud. https://www.who.int/maternal_child_ adolescent/documents/fch_cah_00_7/es/

Pallavicini. (2008). Los objetos de consumo en la contrucción de los procesos de individuación de adolescentes. Última década, 16(29), 29-46. http://dx.doi. org/10.4067/S0718-22362008000200003

Retuerto, A. (2002). Desarrollo del razonamiento moral, razonamiento moral prosocial y empatía en la adolescencia y juventud. Tesis doctoral, Universidad de Valencia, Obtenido de Tesis doctirales en red: http://www.tdx.cat/handle/10803/10205

Ricoeur, P. (2002). Del texto a la acción ensayos de hermenéutica II. México: Fondo de cultura económica.

Suárez, C., Del Moral, G., Musitu. (2013). Medios de comunicación y consumo de alcohol en adolescentes ¿qué dicen los expertos? Salud y drogas, 13 (2), 99-108. http://www.redalyc.org/articulo.oa?id=83929573002

Uribe, A., Sanabria, A., Orcasita, L., Barreto, J. (2016). Conducta antisocial y delictiva en adolescentes y jóvenes colombianos. Informes Psicológicos, 16(2), 103-119. DOI: http://dx.doi.org/10.18566/infpsicv16n2a07

Varela, A., Salazar, I., Cáceres, D., Tovar, R. (2007). Consumo de sustancias psicoactivas ilegales en jóvenes: factores psicosociales asociados. Pensamiento Psicológico, 3(8), 31-45, https://www.redalyc.org/pdf/801/80130804.pdf. 